\title{
DNA Polymerase $\beta$ : Multiple Conformational Changes in the Mechanism of Catalysis $^{\dagger}$
}

\author{
Xuejun Zhong, ${ }^{\ddagger}$ Smita S. Patel, ${ }^{\S, \|}$ Brian G. Werneburg, ${ }^{\ddagger}$ and Ming-Daw Tsai*,\$,§,\| \\ Departments of Chemistry and Biochemistry, The Ohio State University, Columbus, Ohio 43210 \\ Received December 27, 1996; Revised Manuscript Received May 22, $1997^{\otimes}$
}

\begin{abstract}
Stopped-flow fluorescence assay was applied to identify conformational changes in the catalytic cycle of DNA polymerase $\beta$ ( $\mathrm{Pol} \beta$ ), using a synthetic DNA primer/template containing 2-aminopurine (2-AP) at the template position opposite the incoming dNTP. Two phases of fluorescence change were observed in the stopped-flow fluorescence assay of the incorporation of the correct nucleotide dTTP. The rate of the slow phase corresponds to that of product formation. This slow phase was identified as the result of a rate-limiting conformational change step before chemistry because this slow phase was also observed with a dideoxynucleotide at the $3^{\prime}$ end of the primer which prevents chemical bond formation. The fast phase was also attributed to a conformational change step since its dependence on [dTTP] is hyperbolic. The rates of the two phases and their dependence on [dTTP] and $\left[\mathrm{Mg}^{2+}\right]$ suggest that the fast conformational change is induced by the binding of MgdNTP and the slow conformational change is induced by the binding of the catalytic $\mathrm{Mg}^{2+}$ ion. The same biphasic kinetics with different rates were also observed with the thio analog dTTP $\alpha$ S and incorrect nucleotides dATP, dGTP, and dCTP. The structural nature for the two conformational changes has been discussed in relation to the available structural information of this enzyme. The results could help to explain how a polymerase controls and achieves its fidelity with a multiple conformational change mechanism.
\end{abstract}

DNA polymerases catalyze nucleotidyl transfer reactions in a remarkable way in that they display both broad substrate specificity (incorporate four different nucleotides as substrates) and strict substrate specificity (incorporate one correct nucleotide during each catalytic cycle according to the DNA template) (Johnson, 1993). How these enzymes faithfully alternate their dNTP substrates during each catalytic cycle remains to be established at the molecular level. The 1-3 $\mathrm{kcal} / \mathrm{mol}$ free energy difference between correct and incorrect base pairing (Loeb \& Kunkel, 1982) can only account for 5-150-fold selectivity (Johnson, 1993), while the fidelity of DNA polymerases ranges from 1000 to 100000 (Loeb \& Kunkel, 1982; Echols \& Goodman, 1991). Structural analyses of G:T (Hunter et al., 1987) and A:C (Hunter et al., 1986) mispairs revealed little local perturbation of the helix and no effect on the global conformation of the duplex. A conformational change before the chemical bond formation has been suggested in the mechanisms of several DNA polymerases (Patel et al., 1991; Kuchta et al., 1987; Hsieh et al., 1993). This conformational change step has been proposed to play an important role in enhancing the fidelity of T7 DNA polymerase by an induced-fit mechanism (Wong et al., 1991; Johnson, 1993). Multiple RNA polymerase conformational changes have also been proposed to be

$\dagger$ This work was supported by NIH Grants GM43268 (to M.-D.T.) and GM 51966 (to S.S.P.), by American Cancer Society Grant JFRA 565 (to S.S.P.) and by NIH NRSA Postdoctoral Fellowship GM15973 (to B.G.W.). This is paper 4 in the series DNA polymerase $\beta$. For paper 3, see Kraynov et al. (1997).

* Address correspondence to this author at the Department of Chemistry.

† Department of Chemistry.

$\S$ Department of Biochemistry.

"Also members of Biophysics Program and the Ohio State Biochemistry Program.

${ }^{\otimes}$ Abstract published in Advance ACS Abstracts, September 1, 1997. involved in controlling the fidelity of transcription (Erie et al., 1993).

Mammalian DNA polymerase $\beta(\mathrm{Pol} \beta)^{1}$ is a small (39 $\mathrm{kDa}$ ) and simple (with no associated exonuclease or proofreading activities) enzyme which may serve as a model to study the mechanism of the nucleotidyl transfer reaction and the fidelity of DNA polymerases. Pol $\beta$ consists of an $8-\mathrm{kDa}$ $\mathrm{N}$-terminal domain and a $31-\mathrm{kDa} \mathrm{C}$-terminal polymerase domain. Although an excellent mechanistic model of the chemistry step has been proposed (Pelletier et al., 1994), the kinetic mechanism by which $\operatorname{Pol} \beta$ achieves its substrate specificity and fidelity is yet to be established. Pre-steadystate kinetic analysis (Werneburg et al., 1996; Ahn et al., 1997; Kraynov et al., 1997) and steady-state analysis (Abbott et al., 1988; Beard et al., 1996) have provided important information. However, the radioactive gel assays used in these analyses only measure product formation and cannot detect conformational changes along the reaction pathway.

The relatively high intrinsic fluorescence of 2-aminopurine (2-AP) along with its extreme sensitivity to surrounding environment (Ward et al., 1969; Guest et al., 1991; Bloom et al., 1993, 1994; Hochstrasser et al., 1994; Raney et al., 1994) has been successfully used to detect intermediate species along the reaction pathways of the Klenow Fragment of Pol I and T4 DNA polymerase (Frey et al., 1995). We report here detailed kinetic analyses of rat brain Pol $\beta$ (overexpressed in Escherichia coli) using a DNA substrate containing 2-AP. The results led to the finding of multiple

${ }^{1}$ Abbreviations: Pol $\beta$, DNA polymerase $\beta$; 2-AP, 2-aminopurine nucleotide analog; dNTP, $2^{\prime}$-deoxynucleoside $5^{\prime}$-triphosphate; dNTP $\alpha$ S, $2^{\prime}$-deoxynucleoside $5^{\prime}$-O-(1-thiotriphosphate); ddAMP, $2^{\prime}, 3^{\prime}$-dideoxyadenosine $5^{\prime}$-monophosphate; Tris, 2-amino-2-(hydroxymethyl)-1,3propanediol. 


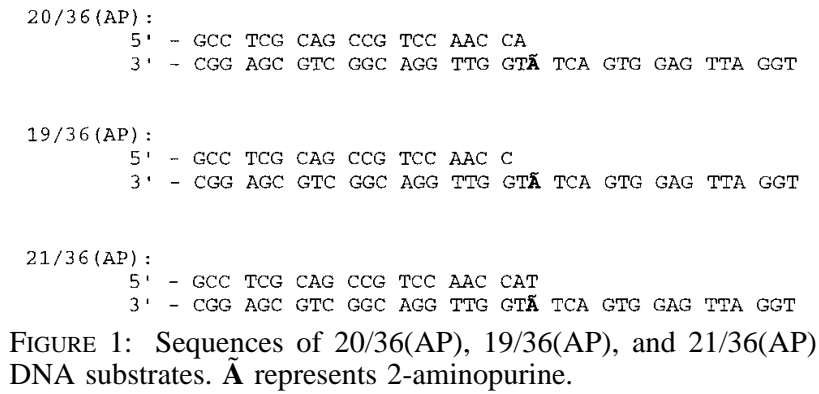

conformational changes and provided insight into how DNA polymerases achieve high fidelity.

\section{MATERIALS AND METHODS}

Nucleotide Triphosphates and Other Materials. All ultrapure dNTPs were purchased from Pharmacia-LKB. $\left[\gamma_{-}{ }^{32} \mathrm{P}\right]-$ ATP and $\left[\alpha-{ }^{32} \mathrm{P}\right] \mathrm{dNTPs}$ were purchased from ICN Biomedicals. dNTP $\alpha$ S were from United States Biochemicals as mixed isomers. T4 polynucleotide kinase was purchased from New England Biolabs. Sep-Pak C18 cartridges were from Millipore Corporation. G-25 microspin columns were from Pharmacia Biotech. All other reagents used were of the highest purity commercially available.

Rat DNA Polymerase $\beta$. Rat DNA polymerase $\beta$ was purified as described (Werneburg et al., 1996). The stock enzyme concentration was determined by $\epsilon_{280}=2.12 \times 10^{4}$ $\mathrm{M}^{-1} \mathrm{~cm}^{-1}$ (Casas-Finet et al., 1991).

DNA Substrates. Synthetic DNA oligodeoxynucleotides were supplied by Integrated DNA Technologies, Inc. The DNA oligonucleotide containing 2-aminopurine was synthesized by the phosphoramidite method. All oligodeoxynucleotide single strands were purified by electrophoresis on $20 \%$ polyacrylamide $-7 \mathrm{M}$ urea denaturing gels. The gel slices were crushed and soaked overnight in $20 \mathrm{~mL} 0.1 \mathrm{M}$ triethylammonium acetate, $\mathrm{pH}$ 7.0, 1 mM EDTA (ethylenediaminetetraacetatic acid) to elute the oligonucleotides. The solutions were then desalted with Sep-Pak C18 cartridges, dried with a speed-vac, and suspended in TE buffer $(10 \mathrm{mM}$ Tris and $0.1 \mathrm{mM}$ EDTA, $\mathrm{pH}$ 8.0). The concentrations of the oligonucleotides were determined by absorbance at 260 nm. DNA stock solutions were made by annealing equal amounts of the primer and the template. The DNA sequences used in this study are listed in Figure 1.

Fluorescence Spectra of 2-AP DNA, Pol $\beta$, Pol $\beta+D N A$, and Pol $\beta+D N A+d T T P$. The emission and excitation spectra of the 2-AP DNA substrate, $\operatorname{Pol} \beta$, Pol $\beta+$ DNA, and Pol $\beta+$ DNA + dTTP were measured. Pol $\beta$ alone had a much higher fluorescence intensity at $345 \mathrm{~nm}$ (maximum emission wavelength for $\operatorname{Pol} \beta$ ) than that for the 2-AP DNA substrate at $375 \mathrm{~nm}$ (maximum emission wavelength for 2-AP) at the same concentration when both were excited at $290 \mathrm{~nm}$. Addition of DNA and/or dTTP to the Pol $\beta$ solution quenched some of the enzyme fluorescence. The maximum excitation wavelengths for the 2-AP DNA substrate and for Pol $\beta$ were 310 and $280 \mathrm{~nm}$, respectively.

Kinetic Assay Buffer. The buffer used in all the kinetic assays was comprised of $50 \mathrm{mM}$ Tris, $50 \mathrm{mM} \mathrm{KCl}$, and $10 \%$ glycerol, $\mathrm{pH} 7.8\left(30^{\circ} \mathrm{C}\right)$. The buffer was degassed before use. All the experiments were conducted at $30^{\circ} \mathrm{C}$.

Stopped-Flow Fluorescence Assay of Polymerization. The experiments were performed with a KinTek stopped-flow spectrometer. The excitation wavelength was $290 \mathrm{~nm}$, and the emission was monitored with a $340 \mathrm{~nm}$ band-pass filter (Corion). Typical assay conditions were as the following: In one syringe, $0.6 \mu \mathrm{M}$ rat DNA polymerase $\beta$ was incubated with $0.6 \mu \mathrm{M}$ 20/36(AP) DNA substrate and $5 \mathrm{mM} \mathrm{Mg}^{2+}$. The second syringe contained $500 \mu \mathrm{M}$ dTTP and $5 \mathrm{mM}$ $\mathrm{Mg}^{2+}$. The reaction was initiated by mixing $80 \mu \mathrm{L}$ solutions from each syringe, and the time course of the fluorescence signal was monitored during the reaction. Multiple experiments were performed, and the data were averaged (9-16 runs) to maximize the signal to noise ratio. Two time windows were used in order to accurately measure both the fast phase and the slow phase. The dTTP concentration dependence experiments were carried out at fixed $5 \mathrm{mM}$ $\mathrm{Mg}^{2+}$. Similarly, the $\mathrm{Mg}^{2+}$ concentration dependence experiments were carried out at fixed $50 \mu \mathrm{M}$ final concentration of dTTP. This dTTP concentration was chosen because it provided the largest fluorescence change for the fast phase. All concentrations were initial concentrations unless otherwise noted.

To measure the kinetics of base misincorporation, 1-2 $\mathrm{mM}$ dNTP concentrations were used. For experiments with $\mathrm{dNTP} \alpha \mathrm{S}$, stock solutions of mixed isomers (1:1) have been used.

Experiments with Other Optical Filters and Other Excitation Wavelengths. Different optical filters were tried to obtain the best signal and information about the source of the fluorescence change. With a $345 \mathrm{~nm}$ cutoff filter, a much larger signal was observed in the stopped-flow fluorescence assay. However, the fluorescence changes of the two phases observed were in the same direction (both decrease), which prevented accurate determination of the rate constants of both phases. With a $360 \mathrm{~nm}$ cutoff filter, similar results have been observed as with the $340 \mathrm{~nm}$ band-pass filter used in this study, but the amplitude of the fast phase was smaller. Excitation at $290 \mathrm{~nm}$ (optimal for tryptophan) resulted in a much stronger signal than at $310 \mathrm{~nm}$ (maximum excitation wavelength for 2-AP).

Rapid Chemical Quench-Flow Assay of Polymerization. Rapid quench-flow assays were conducted under the same conditions as the stopped-flow assays with the specified enzyme and DNA concentrations described in the Results. The primer was $5^{\prime}$-radiolabeled (1000-5000 dpm/pmol) with $\left[\gamma-{ }^{32} \mathrm{P}\right] \mathrm{ATP}$ by $\mathrm{T} 4$ polynucleotide kinase following the manufacturer's protocol. The reactions were performed with an apparatus designed by K. A. Johnson (1986) and built by KinTek Instruments. The reactions were initiated by mixing the two reactants and quenched with $0.5 \mathrm{M}$ EDTA after the reaction time interval. The misincorporation assays were measured manually. Reaction mixtures $(20 \mu \mathrm{L})$ were quenched with $30 \mu \mathrm{L}$ of $0.5 \mathrm{M}$ EDTA, $\mathrm{pH} 8.0$, after the reaction time intervals. The products were separated on $20 \%$ polyacrylamide denaturing gels, and the radioactivity was quantitated with a Beta Scope instrument from Betagen.

Kinetic Simulation. The Penn State version (PSUSIM on the PC, kindly provided by K. A. Johnson at Pennsylvania State University) of the computer program KINSIM (Barshop et al., 1983; Anderson et al., 1988) was used to generate simulation data for possible kinetic schemes. A set of kinetic parameters was first estimated from the real experimental data by curve fittings indicated in the figure legends. Equilibrium constants were used for steps assumed to be in rapid equilibrium (the $\mathrm{dNTP}$ binding step and the $\mathrm{Mg}^{2+}$ 
binding step). Their starting values were estimated as the $K_{\mathrm{m}}$ values obtained from stopped-flow experiments. The starting values for the forward rate constants for the two conformational change steps were estimated as the maximal rates from the stopped-flow experiments. The starting value for the reverse rate constant of the fast conformational change was estimated as the intercept of the plot of $\mathrm{Mg}^{2+}$ concentration dependence of the fast phase. The reverse rate for the slow conformational change was estimated as the intercept of the plot of dTTP concentration dependence of the slow phase. The extinction coefficients for each species were estimated from the intensities of the fluorescence signal. The extinction coefficients for the species in rapid equilibrium were assigned to have the same value. The assignment of the extinction coefficients was relative and arbitrary. However, the rates obtained from the simulation experiments were theoretically independent of the values of the extinction coefficients assigned, and only the amplitude of each species was dependent on the value of the extinction coefficients. This conclusion was also confirmed from the simulation experiments. Once the estimated values of a set of kinetic parameters for a kinetic scheme and the extinction coefficients were chosen, the data were then generated from the computer simulation program PSUSIM at given substrate concentrations and fit to a double exponential to obtain the observed rate constants for the two phases. These observed rates at different substrate concentrations were then compared with the corresponding rates obtained from the real data (the data from the stopped-flow experiments). The kinetic parameters were adjusted manually and the whole process was repeated until the rates from the simulation experiments were as close as possible to the rates from the real data. It was easier to compare the rates rather than the original experimental data because the absolute extinction coefficients for each species were unknown.

\section{RESULTS}

Rapid Chemical Quench-Flow Assay of Incorporation of dTTP Opposite Template 2-Aminopurine. 2-Aminopurine (2-AP) can form a Watson-Crick type base pair with thymine, which is similar to the base pair between adenine and thymine except that the hydrogen bond position is switched from position 6 to 2 of the purine ring. We first measured the rate constant of dTMP addition to a primer opposite to the 2-AP base in the template by a rapid quenchflow assay under the single turnover condition. The sequence of the DNA substrate containing 2-AP, 20/36(AP), is shown in Figure 1. The procedures have been described previously (Werneburg et al., 1996; Ahn et al., 1997). The time course of the reaction using an excess of Pol $\beta$ over the DNA substrate is shown in Figure 2. The data were fit to a single exponential function to give a single nucleotide incorporation rate constant of $5.9 \pm 0.3 \mathrm{~s}^{-1}$ at $250 \mu \mathrm{M}$ dTTP at $30{ }^{\circ} \mathrm{C}$. This observed rate constant of dTMP incorporation opposite $2-\mathrm{AP}$ is within the range of the rate constants of correct dNMP incorporation into a series of $25 / 45$ mer DNA substrates (3.4-24 s ${ }^{-1}$ at $37^{\circ} \mathrm{C}$ ) (Ahn et al., 1997; Kraynov et al., 1997).

Stopped-Flow Fluorescence Assay of Incorporation of dTTP Opposite Template 2-Aminopurine. The reaction carried out using rapid quench-flow experiment was then monitored by stopped-flow fluorescence. A 1:1 stoichiometry for E:DNA was chosen to maximize $\Delta I_{\mathrm{F}} / I_{\mathrm{F}}$ ratio and



FIGURE 2: Incorporation of dTTP opposite template 2-AP as determined by rapid quench-flow assay. A solution of excess $\mathrm{Pol}$ $\beta(2 \mu \mathrm{M})$ preincubated with $5^{\prime}-{ }^{32} \mathrm{P}$ labeled 20/36(AP) DNA substrate $(0.2 \mu \mathrm{M})$, and $5 \mathrm{mM} \mathrm{MgCl}_{2}$ was mixed with equal volume of 500 $\mu \mathrm{M}$ dTTP and $5 \mathrm{mM} \mathrm{MgCl}$ to initiate the reaction. The reactions were quenched with $0.5 \mathrm{M}$ EDTA. The products were separated and quantitated as described in the Experimental Procedures. Concentrations are initial concentrations unless otherwise noted. All reactions were performed at $30^{\circ} \mathrm{C}$ unless otherwise noted. The data were fit to a single exponential with a observed rate constant of $5.9 \pm 0.3 \mathrm{~s}^{-1}$.

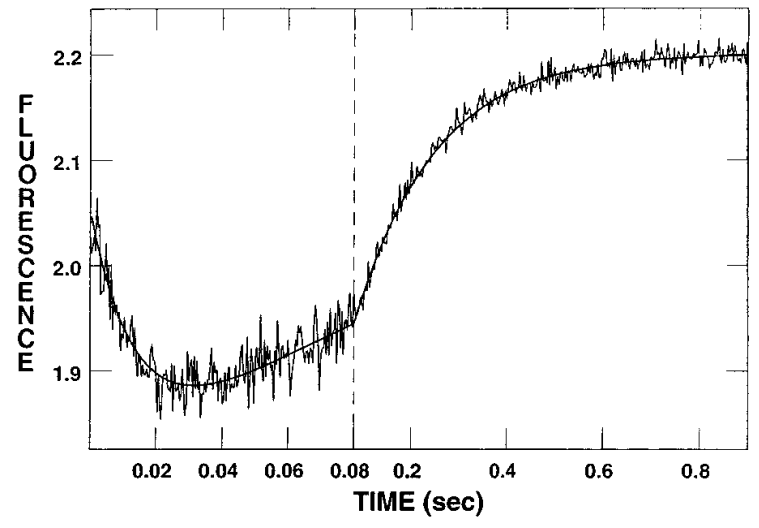

FIGURE 3: Stopped-flow fluorescence assay of incorporation of dTTP opposite template 2-aminopurine. In one syringe, $0.6 \mu \mathrm{M}$ Pol $\beta$ was incubated with $0.6 \mu \mathrm{M} 20 / 36$ (AP) substrate and $5 \mathrm{mM}$ $\mathrm{Mg}^{2+}$. The other syringe contained $500 \mu \mathrm{M}$ dTTP and $5 \mathrm{mM} \mathrm{Mg}^{2+}$. The reaction was initiated by mixing $80 \mu \mathrm{L}$ solutions from each syringe and the fluorescence signal was monitored during the reaction. Multiple experiments were collected and averaged (916 runs) to improve the signal to noise ratio. Two time windows were used to accurately measure the rates of both the fast and the slow phases. The data were fit to a double exponential to yield the observed rate constants of both phases $\left(70 \pm 3\right.$ and $\left.6.0 \pm 0.1 \mathrm{~s}^{-1}\right)$.

$S / N$ ratio in the stopped-flow experiments. As shown in Figure 3, two phases of fluorescence changes were observed upon mixing dTTP with the Pol $\beta$-DNA complex. The data fit well to a double exponential to give two rate constants. The observed rate constant of the slow phase $\left(6.0 \pm 0.1 \mathrm{~s}^{-1}\right.$ at $250 \mu \mathrm{M} \mathrm{dTTP}$ ) is identical to the rate constant of dTTP incorporation determined in the single-turnover rapid quenchflow experiments. The rate of the fast phase $\left(70 \pm 3 \mathrm{~s}^{-1}\right)$ is ca. 12 times faster than that of the slow phase. Similar biphasic stopped-flow kinetics was observed when intrinsic protein fluorescence changes were monitored with the natural adenine-containing DNA substrate, except that the amplitudes of the protein fluorescence changes were much smaller (data not shown).

The two phases could be caused by different microscopic events: binding of dNTP, base pair formation, $\mathrm{Mg}^{2+}$ binding, 

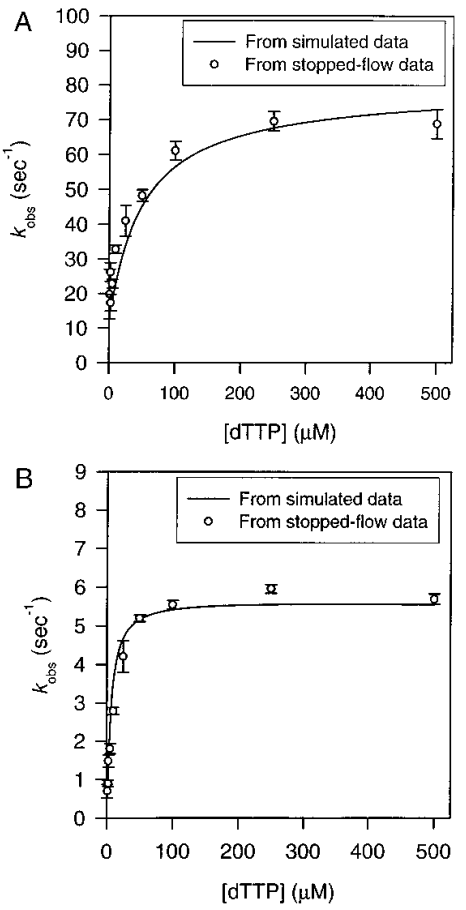

FigURE 4: The dTTP concentration dependence of the observed rate constants for the fast phase (A) and the slow phase (B) at 5 $\mathrm{mM} \mathrm{Mg}{ }^{2+}$. The DNA template/primer used was 20/36(AP). The circles represent the observed rate constants obtained from stoppedflow fluorescence experiments as shown in Figure 3. The data in panel A were fit to the equation $k_{\text {obs }}=k_{\text {int }}+\left(k_{\max }-k_{\text {int }}\right)[\mathrm{dTTP}] /$ $\left(K_{\mathrm{m}}+\right.$ [dTTP] $)$ to give $k_{\max }=75 \pm 5 \mathrm{~s}^{-1}, K_{\mathrm{m}}=41 \pm 13 \mu \mathrm{M}$, and $k_{\text {int }}=19 \pm 3 \mathrm{~s}^{-1}$. The line represents the rate constants (connected with a smooth curve) obtained by fitting the data from the simulation experiments with Scheme 1 and the kinetic parameters in Table 1. The data in panel B were fit to the same equation to yield $k_{\max }=6.1 \pm 0.2 \mathrm{~s}^{-1}, K_{\mathrm{m}}=13 \pm 2 \mu \mathrm{M}$, and $k_{\text {int }}=0.3 \pm 0.2$ $\mathrm{s}^{-1}$ for the slow phase.

phosphodiester formation, or conformational changes induced by these events. Since the rate of the fast phase is greater than the rate of dTTP incorporation, the fast phase should correspond to a step occurring between dNTP binding and the phosphodiester bond formation. The slow phase could be caused by a rate-limiting conformational change prior to chemistry or by the chemical step itself. Further experiments were then designed to probe the nature of the two phases of fluorescence changes, as described in the following sections.

dTTP Concentration Dependence of the Fast and Slow Phases. The stopped-flow kinetic experiments were carried out at different dTTP concentrations. As shown in Figure 4 , the observed rate constants of both phases show a hyperbolic [dTTP] dependence. The data were fit to the following hyperbolic equation:

$$
k_{\text {obs }}=k_{\text {int }}+\left(k_{\max }-k_{\text {int }}\right)[\mathrm{dTTP}] /\left(K_{\mathrm{m}}+[\mathrm{dTTP}]\right)
$$

where $k_{\text {int }}=$ intercept, $k_{\max }=$ maximum rate, and $K_{\mathrm{m}}=$ apparent $K_{\mathrm{d}}$ of dTTP. The results gave $k_{\max }=75 \pm 5 \mathrm{~s}^{-1}$, $K_{\mathrm{m}}=41 \pm 13 \mu \mathrm{M}$, and $k_{\text {int }}=19 \pm 3 \mathrm{~s}^{-1}$ for the fast phase and $k_{\max }=6.1 \pm 0.2 \mathrm{~s}^{-1}, K_{\mathrm{m}}=13 \pm 2 \mu \mathrm{M}$, and $k_{\mathrm{int}}=0.31$ $\pm 0.18 \mathrm{~s}^{-1}$ for the slow phase.

If the fast phase results from the bimolecular process of dTTP binding, the observed rate constant should increase linearly with [dTTP] as discussed in the review article with examples by Johnson (1992). Since the [dTTP] dependence is hyperbolic as shown in Figure 4A, the fast phase is likely

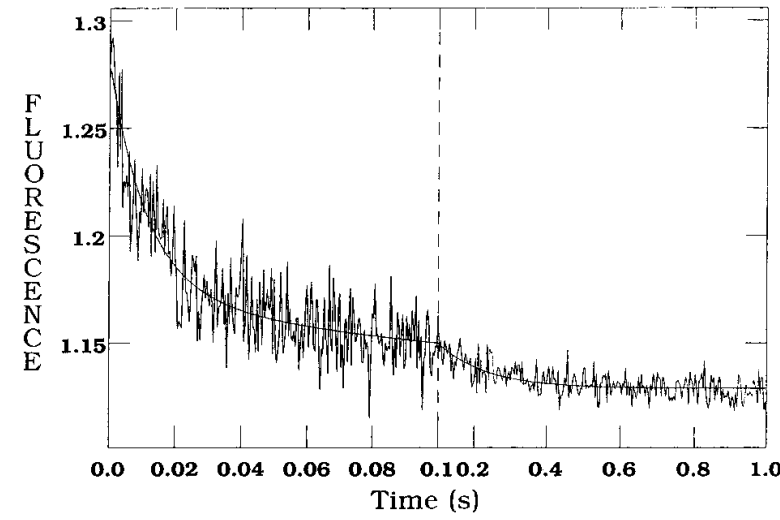

FIGURE 5: Stopped-flow fluorescence assay of the incorporation of dTTP opposite template 2-aminopurine with ddAMP at 3' end of the primer. In one syringe, $0.6 \mu \mathrm{M} \mathrm{Pol} \beta$ was preincubated with $0.6 \mu \mathrm{M}$ 19/36(AP), $50 \mu \mathrm{M}$ ddATP, and $5 \mathrm{mM} \mathrm{Mg}^{2+}$ for $10 \mathrm{~min}$ to incorporate ddAMP. The reaction was initiated by mixing an equal volume of solution from another syringe containing $500 \mu \mathrm{M}$ dTTP and $5 \mathrm{mM} \mathrm{Mg}{ }^{2+}$. The data were best fit to a double exponential which gave the observed rate constants $(87 \pm 8$ and $7.4 \pm 1.2$ $\left.\mathrm{s}^{-1}\right)$

to result from a conformational change induced by dTTP binding, rather than the dTTP binding process. The dTTP dependence, however, cannot resolve whether the slow phase results from a conformational change or the chemical reaction or a combination of them.

Stopped-Flow Fluorescence Assay of Incorporation of dTTP Opposite Template 2-Aminopurine with ddAMP at the $3^{\prime}$ End of the Primer. To dissect the chemical step from a potential conformational change step, we carried out the stopped-flow experiments with a DNA substrate that contains ddAMP at the $3^{\prime}$ end of the primer, thus lacking the $3^{\prime}-\mathrm{OH}$ required for the chemistry to occur. The experiment was carried out by preincubating in one syringe Pol $\beta, 19 / 36-$ (AP), ddATP, and $\mathrm{Mg}^{2+}$ to allow ddAMP to be incorporated into the primer. The rate of ddAMP incorporation was similar to that of dAMP incorporation (data not shown). The stopped-flow reaction was then initiated by adding dTTP and $\mathrm{Mg}^{2+}$ from a second syringe. As shown in Figure 5, two phases of fluorescence changes were observed. Both the fluorescence change and the rate for the fast phase (decrease with a rate of $87 \pm 8 \mathrm{~s}^{-1}$ ) were similar to that observed with the $3^{\prime}-\mathrm{OH}$ containing DNA substrate (decrease with a rate of $70 \pm 3 \mathrm{~s}^{-1}$ ) shown in Figure 3. However, the fluorescence change in the slow phase of Figure 5 showed a decrease with time instead of an increase in Figure 3. Despite the difference in fluorescence change, the rates of the slow phases were comparable $\left(7.4 \pm 1.2 \mathrm{~s}^{-1}\right.$ for ddAMP primer and $6.0 \pm 0.1 \mathrm{~s}^{-1}$ for $3^{\prime}-\mathrm{OH}$ containing primer), which suggests that they are dominated by the same rate-limiting step. The dNTP concentration dependence of the two phases could not be measured with the dideoxy-terminated primer due to the weaker fluorescence signal and the large error associated with the measurement.

Although the rate of the slow phase corresponded to the rate of catalysis, the above results suggest that the slow phase results from a rate-limiting conformational change step prior to chemistry rather than the chemical step itself, since the chemical bond formation was prevented with ddAMP at the $3^{\prime}$ end of the primer. However, it is unclear why the fluorescence signal decreased in the slow phase of Figure 5 but showed an increase in the slow phase of Figure 3. A 


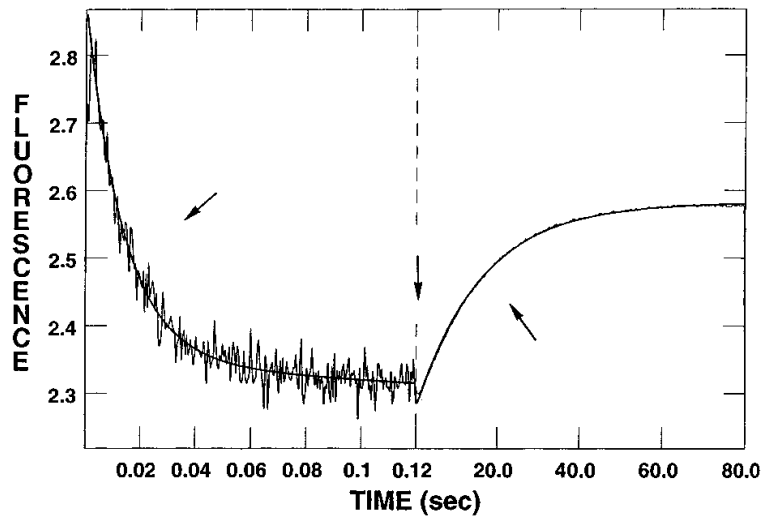

FIGURE 6: Stopped-flow fluorescence assay of the reverse polymerization reaction. In one syringe, $0.6 \mu \mathrm{M} \mathrm{Pol} \beta$ was incubated with $0.6 \mu \mathrm{M} 21 / 36(\mathrm{AP})$ substrate and $20 \mathrm{mM} \mathrm{Mg}^{2+}$. The reaction was initiated by mixing an equal volume of solution from another syringe containing $5 \mathrm{mM} \mathrm{PP}$. The data were best fit to a triple exponential with the observed rate constants of $72 \pm 3,6 \pm 2$, and $0.062 \pm 0.002 \mathrm{~s}^{-1}$. The arrows indicate the three phases detected. The amplitude of the middle phase was small.

possible explanation is that the fluorescence increase for the slow phase in Figure 3 comes from the net fluorescence change of a combination of the rate-limiting conformational change step and additional fast steps that occur after chemistry. These additional steps were not detected as separate phases in Figure 3 since their rates are faster than the rate-limiting conformational change, but large fluorescence increase at these steps contributed to the net fluorescence increase in the slow phase. This interpretation was further tested by the stopped-flow fluorescence experiments in the reverse reaction.

Fluorescence Changes in the Reverse Reaction. The reverse reaction was initiated by mixing $0.6 \mu \mathrm{M}$ Pol $\beta$ preincubated with $0.6 \mu \mathrm{M} 21 / 36(\mathrm{AP})$ substrate and $20 \mathrm{mM}$ $\mathrm{Mg}^{2+}$ from one syringe with equal volume of solution from another syringe containing $5 \mathrm{mM} \mathrm{PP}$. As shown in Figure 6 , three phases of fluorescence changes were detected in the reverse reaction. The rate of the slow phase $(0.062 \pm 0.002$ $\mathrm{s}^{-1}$ ) corresponds to the rate of dNTP product formation as determined from the rapid quench-flow experiment using radiolabeled DNA (data not shown). If this phase results from the chemical reaction, then the other two phases should result from steps before the chemical reaction in the reverse direction. If the slow phase results from a rate-limiting conformational change prior to the chemical step in the reverse direction, then all three phases should be caused by steps prior to the chemical step in the reverse direction. In either case, the results suggest that in the forward direction there are additional fluorescence changes from the chemical step and after, which could explain the difference in the fluorescence change of the slow phase between Figure 3 and Figure 5. The nature of the three phases in the reverse reaction, however, remains to be established by future studies.

$\mathrm{Mg}^{2+}$ Concentration Dependence of the Fast and Slow Phases. The X-ray crystal structure of the ternary complex of Pol $\beta$, DNA, and ddCTP has revealed that two $\mathrm{Mg}^{2+}$ ions bind to the substrates and the active site residues (Pelletier et al., 1994, 1996a,b). One $\mathrm{Mg}^{2+}$ is located at the dNTP binding site where it binds to the $\beta, \gamma$-phosphates of dNTP and the carboxylates of Asp190 and Asp192. The second $\mathrm{Mg}^{2+}$ binds to the $\alpha$-phosphate of dNTP and the $3^{\prime}-\mathrm{OH}$ of the primer and the carboxylates of Asp256, Asp190, and
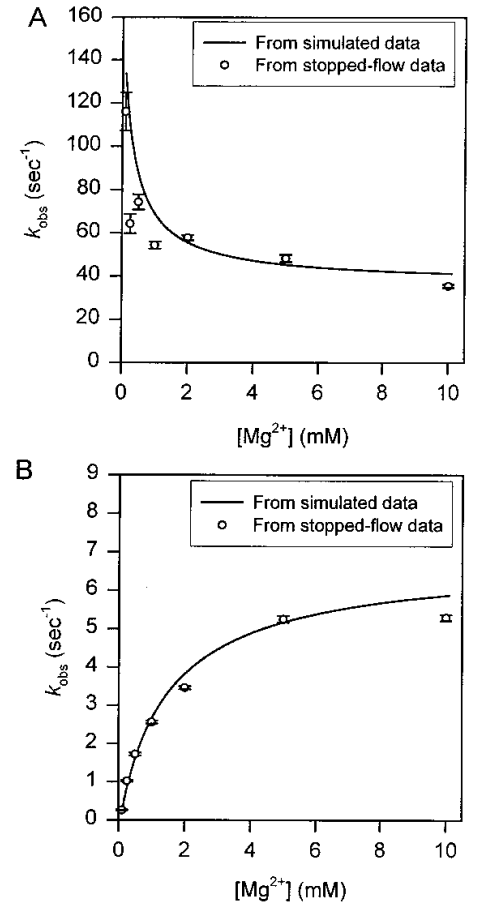

FIGURE 7: $\mathrm{Mg}^{2+}$ concentration dependence of the observed rate constants for the fast phase (A) and the slow phase (B) at $50 \mu \mathrm{M}$ dTTP. The DNA template/primer used was 20/36(AP). The circles represent the observed rate constants obtained from stopped-flow fluorescence experiments. The line represents the rate constants (connected with smooth curve) obtained by fitting the data from the simulation experiments with Scheme 1 and kinetic parameters in Table 1. The data in panel B were fit to the equation $k_{\mathrm{obs}}=k_{\max }$ $\left[\mathrm{Mg}^{2+}\right] /\left(K_{\mathrm{m}}+\left[\mathrm{Mg}^{2+}\right]\right)$ to give $k_{\max }=6.1 \pm 0.2 \mathrm{~s}^{-1}$ and $K_{\mathrm{m}}=1.4$ $\pm 0.2 \mathrm{mM}$.

Asp192. However, very little is known about the kinetics of $\mathrm{Mg}^{2+}$ or MgdNTP binding steps. Stopped-flow experiments were carried out at increasing $\mathrm{Mg}^{2+}$ concentrations, and the $\left[\mathrm{Mg}^{2+}\right]$ dependence of the $k_{\mathrm{obs}}$ values of the fast and the slow phases is shown in Figure 7. The [dTTP] was fixed at $50 \mu \mathrm{M}$ in these experiments since at higher dTTP concentration part of the signal for the fast phase was lost in the mixing time due to faster rates. Interestingly, the fast and slow phases showed different dependence on the $\mathrm{Mg}^{2+}$ concentration. The observed rate constant of the fast phase decreased with $\left[\mathrm{Mg}^{2+}\right]$ (Figure 7A) while that of the slow phase increased with $\left[\mathrm{Mg}^{2+}\right]$ (Figure $7 \mathrm{~B}$ ). The $\left[\mathrm{Mg}^{2+}\right]$ dependence of the slow phase can be fit to a hyperbolic equation to give $k_{\max }=6.1 \pm 0.2 \mathrm{~s}^{-1}$ and $K_{\mathrm{m}}=1.4 \pm 0.2$ $\mathrm{mM}$, where $k_{\max }=$ maximum rate and $K_{\mathrm{m}}=$ apparent $K_{\mathrm{d}}$ of $\mathrm{Mg}^{2+}$.

The hyperbolic dependence of the slow phase on $\left[\mathrm{Mg}^{2+}\right]$ suggests that the slow conformational change occurs after $\mathrm{Mg}^{2+}$ binding. We postulate that this is the second $\mathrm{Mg}^{2+}$ ion, i.e., the catalytic $\mathrm{Mg}^{2+}$. $\mathrm{The}^{2+} \mathrm{Mg}^{2+}$ ion that coordinates with the $\beta, \gamma$-phosphates of dNTP should be considered as being associated with the nucleotide since this metal ion has never been observed in Pol $\beta$ crystal structures except when accompanied by a nucleotide or pyrophosphate (Pelletier et al., 1994, 1996b).

The $\mathrm{Mg}^{2+}$ dependence for the fast phase as shown in Figure 7A could not be readily explained. However, it can be explained by the result of kinetic simulations discussed below. 
Kinetic Model of DNA Polymerase $\beta$. The results and interpretations described in the previous sections led us to propose the following minimal kinetic model (Scheme 1) for Pol $\beta$ up to the chemical step:

Scheme 1

$$
\begin{aligned}
\mathrm{E} \cdot \mathrm{D}_{n} \underset{k_{-1}}{\stackrel{k_{1}[\mathrm{~N}]}{\rightleftharpoons}} \mathrm{E} \cdot \mathrm{D}_{n} \cdot \mathrm{N} \underset{k_{-2}}{\stackrel{k_{2}}{\rightleftharpoons}} \mathrm{E}^{\prime} \cdot \mathrm{D}_{n} \cdot \mathrm{N} \underset{k_{-3}}{\stackrel{k_{3}[\mathrm{M}]}{\rightleftharpoons}} \\
\mathrm{E}^{\prime} \cdot \mathrm{D}_{n} \cdot \mathrm{N} \cdot \mathrm{M} \underset{k_{-4}}{\stackrel{k_{4}}{\rightleftharpoons}} \mathrm{E}^{\prime \prime} \cdot \mathrm{D}_{n} \cdot \mathrm{N} \cdot \mathrm{M} \underset{k_{-5}}{\stackrel{k_{5}}{\rightleftharpoons}} \mathrm{E}^{\prime \prime} \cdot \mathrm{D}_{n+1} \cdot \mathrm{P} \cdot \mathrm{M}
\end{aligned}
$$

where $\mathrm{E}, \mathrm{D}, \mathrm{N}, \mathrm{M}$, and $\mathrm{P}$ represent Pol $\beta$, DNA template/ primer, deoxynucleoside triphosphate $-\mathrm{Mg}^{2+}$ complex (MgdNTP), catalytic $\mathrm{Mg}^{2+}$ ion, and inorganic pyrophosphate, respectively. In this model, Pol $\beta$ undergoes two conformational changes (step 2 and step 4) prior to the chemical step. The first conformational change is induced by MgdNTP binding (step 1) while the second conformational change is induced by catalytic $\mathrm{Mg}^{2+}$ binding (step 3 ).

It is important to note that the experimental data alone would not be sufficient to support Scheme 1. An uncertainty, for example, is whether binding of the catalytic $\mathrm{Mg}^{2+}$ ion occurs both before and after the fast conformational change or only after. However, kinetic simulation of the experimental data, as described in the next section, supports Scheme 1 as the kinetic model that best explains all the experimental results.

Kinetic Simulation of the Stopped-Flow Fluorescence Data. Kinetic simulations were performed with the Penn State version (PSUSIM) of the computer program KINSIM (Barshop et al., 1983; Anderson et al., 1988). Scheme 1 and its kinetically equivalent Scheme 2 (confirmed from computer simulations, also refer to the appendix) stipulate that the binding of the catalytic $\mathrm{Mg}^{2+}$ can only occur after the fast conformational change and before the slow conformational change. Scheme 3 stipulates that the binding of the catalytic $\mathrm{Mg}^{2+}$ can occur both before and after the fast conformational change. Both require binding of the catalytic $\mathrm{Mg}^{2+}$ to induce the slow conformational change.

Scheme 2

$$
\begin{aligned}
& \mathrm{E} \cdot \mathrm{D}_{n} \underset{k_{-1}}{\stackrel{k_{1}[\mathrm{~N}]}{\rightleftharpoons}} \mathrm{E} \cdot \mathrm{D}_{n} \cdot \mathrm{N} \underset{k_{-2} K_{\mathrm{d}, \mathrm{M}} /\left(\mathrm{K}_{\mathrm{d}, \mathrm{M}}+[\mathrm{M}]\right)}{\rightleftharpoons} \\
& \mathrm{E}^{\prime} \cdot \mathrm{D}_{n} \cdot \mathrm{N} \stackrel{k_{4}[\mathrm{M}] /\left(K_{\mathrm{d}, \mathrm{M}}+[\mathrm{M}]\right)}{=} \mathrm{E}_{-4}{ }^{\prime \prime} \cdot \mathrm{D}_{n} \cdot \mathrm{N}
\end{aligned}
$$

Scheme 3

$$
\mathrm{E} \cdot \mathrm{D}_{n} \underset{k_{-1}}{\stackrel{k_{1}[\mathrm{~N}]}{\rightleftharpoons}} \mathrm{E} \cdot \mathrm{D}_{n} \cdot \mathrm{N} \underset{k_{-2}}{\stackrel{k_{2}}{\rightleftharpoons}} \mathrm{E}^{\prime} \cdot \mathrm{D}_{n} \cdot \mathrm{N} \underset{k_{-4}}{\stackrel{k_{4}[\mathrm{M}] /\left(K_{\mathrm{d}, \mathrm{M}}+[\mathrm{M}]\right)}{\rightleftharpoons}} \mathrm{E}^{\prime \prime} \cdot \mathrm{D}_{n} \cdot \mathrm{N}
$$

Kinetic simulation with Scheme 3 showed slight increase of the observed rate of the fast phase with increasing $\mathrm{Mg}^{2+}$ ion concentration, which does not agree with the experimental data. On the other hand, kinetic simulation demonstrated that the experimental data fit well to Scheme 1 (Figures 4,7). These results suggest that binding of the catalytic $\mathrm{Mg}^{2+}$ occurs only after the initial conformational change as indicated in Scheme 1 or at least the $E \cdot D \cdot N$ ternary complex has much higher affinity toward $\mathrm{Mg}^{2+}$ after the initial conformational change.

The chemical step in Scheme 1 is fast and could not be detected as a separate step; therefore, it was not considered
Table 1. Kinetic Parameters in the Mechanism of DNA Polymerase $\beta^{a, b}$

$$
\begin{array}{ll}
\hline \mathrm{E} \cdot \mathrm{D}_{n} \underset{k_{-1}}{\stackrel{k_{1}[\mathrm{~N}]}{\rightleftharpoons}} \mathrm{E} \cdot \mathrm{D}_{n} \cdot \mathrm{N} & K_{\mathrm{d}, \mathrm{N}}=45 \pm 11 \mu \mathrm{M} \\
\mathrm{E} \cdot \mathrm{D}_{n} \cdot \mathrm{N} \underset{k_{-2}}{\stackrel{k_{2}}{\rightleftharpoons}} \mathrm{E}^{\prime} \cdot \mathrm{D}_{n} \cdot \mathrm{N} & k_{2}=70 \pm 7 \mathrm{~s}^{-1}, k_{-2}=110 \pm 40 \mathrm{~s}^{-1} \\
\mathrm{E}^{\prime} \cdot \mathrm{D}_{n} \cdot \mathrm{N} \underset{k_{-3}}{\stackrel{k_{2}[\mathrm{M}]}{\rightleftharpoons}} \mathrm{E}^{\prime} \cdot \mathrm{D}_{n} \cdot \mathrm{N} \cdot \mathrm{M} & K_{\mathrm{d}, \mathrm{M}}=0.35 \pm 0.04 \mathrm{mM} \\
\mathrm{E}^{\prime} \cdot \mathrm{D}_{n} \cdot \mathrm{N} \cdot \mathrm{M} \underset{k_{-4}}{\stackrel{k_{4}}{\rightleftharpoons}} \mathrm{E}^{\prime \prime} \cdot \mathrm{D}_{n} \cdot \mathrm{N} \cdot \mathrm{M} & \left.k_{4}=68 \pm 0.4 \mathrm{~s}^{-1}, \underset{(k-4}{<}<0.10 \pm 0.08 \mathrm{~s}^{-1}\right)^{c} \\
\mathrm{E}^{\prime \prime} \cdot \mathrm{D}_{n} \cdot \mathrm{N} \cdot \mathrm{M} \underset{k_{-5}}{\stackrel{k_{5}}{\rightleftharpoons}} \mathrm{E}^{\prime \prime} \cdot \mathrm{D}_{n+1} \cdot \mathrm{P} \cdot \mathrm{M} & \left(k_{5}>k_{4}\right)^{d}
\end{array}
$$

${ }^{a} \mathrm{E}, \mathrm{D}, \mathrm{N}, \mathrm{M}$, and $\mathrm{P}$ represent rat DNA polymerase $\beta$, DNA template/ primer, deoxynucleoside triphosphate- $\mathrm{Mg}^{2+}$ complex (MgdNTP), catalytic $\mathrm{Mg}^{2+}$ ion, and inorganic phosphate, respectively. ${ }^{b}$ The standard deviation of each kinetic parameter was estimated with the analytical solution given in the appendix and the SigmaPlot program. Searching the optimal parameters directly with SigmaPlot failed to converge. Therefore, the whole optimal parameter set was obtained manually by visual comparison and the standard deviation of each parameter was estimated by varying only one parameter at a time. Each data point was weighted according to its experimental error. ${ }^{c} k-4$ represents a combination of the reverse rate constant of step 4 and the equilibrium constants of the steps afterward. The favorable equilibrium toward final product release results in the backward reaction to be negligible. ${ }^{d}$ The chemical step is fast and was not considered as a separate step during kinetic simulation.

as a separate step during computer simulation. The MgdNTP and the catalytic $\mathrm{Mg}^{2+}$ binding steps were assumed to be rapid equilibrium steps and only equilibrium constants were used in the simulation. It was assumed that $[\mathrm{MgdNTP}]=$ $[\mathrm{dNTP}]_{\text {total. }}$. The kinetic constants were obtained by adjusting each of the intrinsic rate constants manually until the simulated kinetics fit the observed kinetics. This was repeated at all dTTP and $\mathrm{Mg}^{2+}$ ion concentrations until one set of rate constants was found to globally fit all the observed kinetics. The kinetic constants obtained from simulation are listed in Table 1.

The $\mathrm{Mg}^{2+}$ concentration dependence of the fast phase can be easily understood from the approximate solution for $k_{\mathrm{obs}, 1}$ (see Appendix).

The first and the last terms are independent of $\left[\mathrm{Mg}^{2+}\right]$. The second term decrease from the value of $k_{-2}$ to zero with increasing $\left[\mathrm{Mg}^{2+}\right]$ and the third term increase from zero to $k_{4}$ with increasing $\left[\mathrm{Mg}^{2+}\right]$. Since $k_{-2}$ is much greater than $k_{4}$, the overall observed rate decreases with increasing $\left[\mathrm{Mg}^{2+}\right]$.

There were some differences between the simulated results and those from the stopped-flow data at low $\mathrm{Mg}^{2+}$ concentrations in Figure 7A. One possible reason is that not all of dNTP was in the form of MgdNTP at low $\left[\mathrm{Mg}^{2+}\right]$. The dissociation constant of MgATP has been estimated to be from 20 to $300 \mu \mathrm{M}$ (Pecoraro et al., 1984). The above mentioned differences in Figure 7A can be reduced if we assume the dissociation constant of MgdTTP is about 100 to $300 \mu \mathrm{M}$.

Another possible scheme containing the fast conformational change step before dNTP binding was also considered. This was, however, not possible because it would have resulted in a decrease in the observed rate constants of the fast phase with increasing dNTP concentration, which was not observed. 


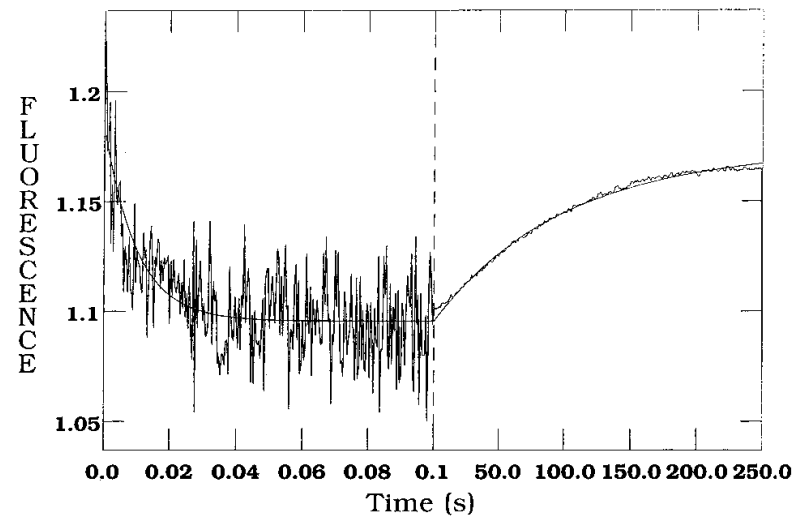

FIGURE 8: Stopped-flow fluorescence assay of misincorporation of dATP opposite template 2-aminopurine. In one syringe, $0.6 \mu \mathrm{M}$ Pol $\beta$ was incubated with $0.6 \mu \mathrm{M} 20 / 36$ (AP) substrate and $5 \mathrm{mM}$ $\mathrm{Mg}^{2+}$. The reaction was initiated by mixing equal volume of solution from another syringe containing $4000 \mu \mathrm{M}$ dATP and 5 $\mathrm{mM} \mathrm{Mg}^{2+}$. The data were fit to a double exponential with observed rate constants of $100 \pm 10$ and $0.010 \pm 0.001 \mathrm{~s}^{-1}$.

Thio Effect for the Incorporation of $d N T P \alpha \mathrm{S}$. To obtain further evidence of the kinetic mechanism, we performed the stopped flow experiments with dTTP $\alpha \mathrm{S}$. The same two phases of fluorescence change were observed, with a thio effect (rate of dTTP/rate of dTTP $\alpha$ S) of 1.5 for the fast phase and 4 for the slow phase. A comparable thio effect (4.3) has been observed in the rapid quench-flow assay (Werneburg et al., 1996). Since we used mixed isomers of dNTP $\alpha \mathrm{S}^{2}$ the above thio effect could be lowered to half of the values shown if we assume that the two isomers have the same binding affinities for the initial dNTP binding step. The small thio effect observed in both the rapid quench-flow and the stopped-flow fluorescence assays is consistent with the suggestion that the slow conformational change is the rate-limiting step.

Stopped-Flow Fluorescence Assay of Misincorporation of dNTP Opposite Template 2-Aminopurine. As shown above, the stopped-flow assay provides an accurate and convenient method to follow the conformational changes during DNA synthesis. One of the important questions related to polymerases is its mechanism of fidelity. To better understand the mechanism by which pol $\beta$ discriminates against incorrect nucleotides, we investigate here the stopped-flow fluorescence changes associated with the incorporation of incorrect nucleotides. The experiments were conducted in the same way as the incorporation of correct dTTP, except that the experiments were carried out at much higher dNTP concentrations.

The stopped-flow fluorescence changes for dATP misincorporation are shown in Figure 8. The same biphasic kinetics, as with the correct nucleotide addition, was observed. The observed rate constant of the fast phase was $100 \pm 9.9 \mathrm{~s}^{-1}$ at $2 \mathrm{mM}$ dATP, which is comparable to the observed rate constant with the correct nucleotide, dTTP, incorporation. The observed rate constant of the slow phase was $0.01 \pm 0.001 \mathrm{~s}^{-1}$, which is about 600 -fold slower than that with the correct nucleotide. This rate also corresponds to the rate of product formation, $0.0075 \mathrm{~s}^{-1}$, determined from the chemical quench-flow experiment. Since the amplitudes

${ }^{2}$ The dNTP $\alpha$ S used in this work and in Werneburg et al. (1996) are both mixtures of $R_{p}$ and $S_{p}$ isomers. They were mistakenly stated as $S_{p}$ isomer in Werneburg et al. (1996) due to mislabeling by the vendor. of the fluorescence changes at lower [dATP] were small, we were unable to obtain reliable [dATP] dependence data.

For dGTP, very small signal in the stopped-flow experiment was observed at the final concentration of $4 \mathrm{mM}$ dGTP. The rate of the fast phase was very fast $\left(360 \pm 190 \mathrm{~s}^{-1}\right)$. The rate of the slow phase was very slow (close to the rate of signal bleaching) and could not be determined accurately.

Deoxy-CTP can form a correct Watson-Crick base pair with 2-AP but with only one hydrogen bond. At $2 \mathrm{mM}$ dCTP, the observed rate constant of the fast phase was 34 $\pm 10 \mathrm{~s}^{-1}$, which is slightly slower than that of correct dTTP, and the rate of the slow phase $\left(0.68 \pm 0.06 \mathrm{~s}^{-1}\right)$ was only about 9-fold slower. The rate of the slow phase was comparable to the rate of product formation $\left(0.4 \pm 0.1 \mathrm{~s}^{-1}\right)$.

\section{DISCUSSION}

Identification of Two Conformational Changes between Nucleotide Binding and Chemistry by Stopped-Flow Fluorescence Assays. A conformational change step before chemistry has been proposed in the mechanism of several DNA polymerases (Patel et al., 1991; Hsieh et al., 1993; Kuchta et al., 1987). Using 2-AP as a fluorescence probe, Frey et al. (1995) have successfully decoupled the conformational change and the chemical steps for nucleotide misincorporation by Klenow Fragment of Pol I. In our previous work with Pol $\beta$, we have also suggested that the chemistry step is at best only partially rate-limiting on the basis of small thio effects (Werneburg et al., 1996). However, no direct evidence of a conformational change step for Pol $\beta$ has been reported previously.

In this work, we have used 2-AP as a fluorescence probe to directly observe two phases of fluorescence changes. The nucleotide dependence and $\mathrm{Mg}^{2+}$ dependence of each phase as well as the result with ddAMP substrate suggest that both phases result from conformational changes before the chemical step and that the fast or first conformational change is induced by MgdNTP binding while the slow or second conformational change is induced by the catalytic $\mathrm{Mg}^{2+}$ binding. By using kinetic simulation, the results finally led to the kinetic model for $\operatorname{Pol} \beta$ (Scheme 1).

The observation of two conformational changes between the nucleotide binding and the chemistry step is unprecedented for a DNA polymerase. Two conformational changes were also reported for HIV reverse transcriptase (Rittinger et al., 1995), but at different steps (one induced by binding of template/primer; the other by the nucleotide) from what we observed for $\operatorname{Pol} \beta$. In the following sections, we discuss the possible structural nature of each conformational change and the implication of the two conformational changes in the mechanism of fidelity. However, Scheme 1 should still be considered as a working model and be subjected to fine tuning by further investigation.

It should also be noted that the fluorescence changes do not simply result from quenching of fluorescence upon base pairing to 2-AP. Protein fluorescence changes with the same kinetics were also observed with the normal adenosine DNA substrate, although the amplitudes were much smaller (data not shown). In fact, we believe the observed fluorescence changes originated largely from protein rather than from 2-AP, since these fluorescence changes were larger when the samples were excited at $290 \mathrm{~nm}$ (optimal for tryptophan) rather than at $310 \mathrm{~nm}$ (optimal for 2-AP). The major role 
of 2-AP is probably to enhance the enzyme fluorescence change which may involve fluorescence energy transfer from tryptophan to 2-AP. Pol $\beta$ has only one tryptophan, which is located in the thumb domain (Pelletier et al., 1994). This thumb domain is believed to be involved in the first conformational change as addressed in the next section.

Possible Structural Origin of the First Conformational Change. We postulate that the nucleotide binding step (step 1) in our mechanism is nonspecific and the fast conformational change (step 2 ) is probably associated with base pairing after the initial nonspecific binding. Pol $\beta$ discriminates its substrates through binding by a factor of 5-50 as was shown in the differences of apparent $K_{\mathrm{d}}$ determined from the chemical quench-flow experiments (Ahn et al., 1997). This factor agrees with the base pairing or binding energy differences (Johnson, 1993). Crystallographic evidence also supports the idea that initial binding of nucleotide may be nonselective (non-base-dependent) and occurs primarily via the triphosphate and sugar moieties (Pelletier et al., 1996b). On the basis of the experimental data from X-ray crystallography (Pelletier et al., 1994, 1996a), the fast conformational change identified in this report could be involved in the closing of the thumb domain. The thumb domain is in the closed form in the crystal structure of the ternary complex of rat Pol $\beta$ complexed with a template-primer and ddCTP (Pelletier et al., 1994), while this domain is in the open form in the human Pol $\beta$-DNA binary complex (Pelletier et al., 1996a).

The observed rate constants of the fast phase for incorrect nucleotide incorporation support that the fast conformational change step is associated with base pairing. As compared with the correct base pair, the higher energy level for the incorrect base pair in the energy diagram would increase the rate of base-pair dissociation and decrease the rate of basepair formation at a less extent. If we assume that $k_{2}$ decreases only about $1-2$-fold, then $k_{-2}$ would increase about $2.5-$ 50-fold since the apparent $K_{\mathrm{d}}$ increases about 5-50-fold for Pol $\beta$ (Ahn et al., 1997). With the analytical solution given in the Appendix, the observed rates of the fast phase for incorrect nucleotide incorporation can be estimated to be 50$420 \mathrm{~s}^{-1}$ using the kinetic parameters for correct nucleotide incorporation listed in Table 1 as reference. Therefore, our experimental data were consistent with the predicted values. For dCMP incorporation, since dCMP forms correct type base pair with one H-bonding, the base-pairing energy difference is expected to be less and the observed rate of the fast phase could be lower than $50 \mathrm{~s}^{-1}$.

Possible Structural Origin of the Second Conformational Change. The slow conformational change is likely to be involved in further alignment of the substrates to a geometry ready for chemistry. We postulate that it could be involved in the displacement of the substrates as part of the translocation process. In the crystal structure of human Pol $\beta$ complex with DNA and ddTTP (Beard et al., 1996), the incoming dTTP is displaced $1.2 \AA$ compared with the structure of rat Pol $\beta$ complex with DNA and ddCTP (Pelletier et al., 1994). Although Beard et al. (1996) considered the structure as that of a ternary complex, there is a possibility that it represents a binary complex, that is, one ddTTP has been incorporated into DNA and the next ddTTP has not yet bound. There are three reasons which suggest this possibility. First, ddTTP should be incorporated just like in the rat Pol $\beta$ ternary complex. Second, the $5^{\prime}$ - triphosphate of ddTTP was not observed, further suggesting that the reaction was completed and $\mathrm{PP}_{\mathrm{i}}$ was released. Third, the thumb domain of the structure is in the open form rather than the closed form found in the rat $\operatorname{Pol} \beta$ ternary complex. A possible reason that the next ddTTP did not bind to the human Pol $\beta$ complex may be due to the weaker affinity of ddTTP relative to ddCTP since the apparent $K_{\mathrm{d}}$ of dCTP was about 5 times lower than that of dTTP (Ahn et al., 1996).

If the crystal structure of the human Pol $\beta$ complex reported by Beard et al. (1996) represents a binary complex structure, it is likely that the slow conformational change we observed is involved in further displacement of the primer by about $2 \AA$, which would allow the incoming dNTP from a hanging position to fit into the active site. We think the translocation could occur during polymerization instead of it being a separate process. Before the binding of the next incoming dNTP, the template has translocated $3.8 \AA$ in the human Pol $\beta$ complex compared with the rat Pol $\beta$ ternary complex whereas the primer has translocated only $1.2 \AA$ (Beard et al., 1996). We suggest that binding of dNTP closes the thumb domain and brings the $\alpha$-phosphate of dNTP closer to the $\mathrm{Mg}^{2+}$ ion at the catalytic site which then allows the $\mathrm{Mg}^{2+}$ ion to bind to the $\alpha$-phosphate of dNTP. Binding of the $\mathrm{Mg}^{2+}$ ion to the $\alpha$-phosphate of dNTP drags the dNTP down to the active site which fully translocates the primer. It is expected that the displacement of the primer requires relatively high energy which agrees with the observation that the slow conformational change is the rate-limiting step. After the reaction is completed, the thumb domain opens and results in the full translocation of the template and one third displacement of the primer. At this point, one catalytic cycle has been completed.

Rate-limiting Step. In the previous report (Werneburg et al., 1996), we stated that "the chemical step appears to be only partially rate-limiting" on the basis of the small thio effect in $k_{\text {pol }}$ (4.3), and that "the results are consistent with the existence of a substrate-induced conformational change that is also partially rate-limiting". We have now identified the rate-limiting conformational change by the stopped-flow fluorescence assay with ddAMP terminated primer. The use of thio effect to determine the rate-limiting step may not be reliable for two reasons. (a) The full thio effect for the chemical step is not known. A thio effect as large as 60 has been observed in T7 DNA polymerase (Wong et al., 1991). Our previous assessment that the chemical step appears to be partially rate-limiting was based on the consideration that the full thio effect is in the range 4-11 (Herschlag et al., 1991), which is probably on the low side. (b) Thio effects may also come from nonchemical steps. A thio effect of 36 on DNA binding was observed for KlenowFragment of Pol I (Dahlberg et al., 1991). We have also observed a thio effect of 1.5 for the fast and 4 for the slow phase as described in this paper. Thus, the thio effect in $k_{\text {pol }}$ (4.3) could be fully attributed to the thio effect in the slow conformational change step.

Control of Fidelity by a Multiple Conformational Change Mechanism. The molecular mechanism that DNA polymerases utilize to achieve their fidelity is still not well understood. An induced-fit mechanism was proposed to explain the fidelity of T7 DNA polymerase (Wong et al., 1991). However, it seems hard to explain the additional selectivity provided by the rate-limiting conformational change induced by nucleotide binding with one conforma- 
tional change alone. Fersht $(1974,1985)$ has also argued that an induced-fit mechanism can not contribute any more to selectivity than a simple one-step binding mechanism with the same free energy of binding.

As for Pol $\beta$, the binding of MgdNTP induces the fast conformational change, while the rate-limiting slow conformational change is induced by the binding of the catalytic $\mathrm{Mg}^{2+}$ ion instead of the binding of the nucleotide. On the basis of this new mechanism, we propose a detailed molecular approach for the control of the fidelity of DNA polymerase $\beta$. As we discussed earlier, the fast conformational change is probably associated with base pairing. Discrimination in this step corresponds to the discrimination through apparent $K_{\mathrm{d}}$ and the base-pairing energy differences. Although selection at this fast conformational change step itself is not highly significant, this step provides a molecular basis for the discrimination in the following steps. Correct nucleotide binding induces the fast conformational change, which brings the $\alpha$-phosphate of the dNTP into the right position for the catalytic $\mathrm{Mg}^{2+}$ binding and allows the second slow conformational change to be induced efficiently. However, a bad base-pair geometry could form after the initial conformational change induced by the incorrect nucleotide binding. This improper base-pair geometry deviates the $\alpha$-phosphate of the dNTP from the right position for the catalytic $\mathrm{Mg}^{2+}$ binding, which could greatly raise the kinetic energy barrier for the second slow conformational change. The second slow conformational change is probably involved in proper alignment of the substrates into a geometry ready for chemistry. After the second conformational change, the $\alpha$-phosphate of the dNTP that is incorrectly base paired could continue to deviate from the right position and orientation for the forming of the transition state of the chemical step, which could provide additional selectivity.

In the above model, the geometry of the base pair in the corresponding enzyme active site conformation plays a central role in the control of fidelity. This should be the case for DNA polymerases. It is not possible for DNA polymerases to control fidelity by optimizing interactions with the bases, since they alternate their substrate specificity during each catalytic cycle. The correct nucleotide for the current cycle will be the incorrect one for the next cycles. However, the correct base-pair geometry varies little in each cycle. Since the base-pairing energy difference could only provide 5-150-fold selectivity, more selectivity must come from the difference of the base-pair geometry. The improper base-pair geometry could result in small deviations of the $\alpha$-phosphate of the nucleotide, which could significantly increase the kinetic energy barrier for both the slow conformational change and the chemical step.

It has been suggested that the shape complementarity of the base pair not the hydrogen bonding may be the chief mechanism in the control of fidelity (Moran et al. 1997). When difluorotoluene (a nonpolar isostere for thymine) replaces thymine as a template base, $V_{\max }$ was about same for correct nucleotide incorporation and $K_{\mathrm{m}}$ increased about 6-fold in their steady-state assay for Klenow-Fragment $\left(\mathrm{exo}^{-}\right)$of DNA polymerase I. Fidelity for difluorotoluene is also comparable with thymine. Their results are consistent with our model because difluorotoluene can form correct base-pair geometry with adenine.
The role of hydrogen bonding in fidelity, however, needs to be studied further. The 6-fold increase in $K_{\mathrm{m}}$ for difluorotoluene should be attributed to the loss of hydrogenbonding energy. Although the $V_{\max }$ for difluorotoluene was about same as thymine for correct nucleotide incorporation, the rate-limiting step in their steady state assay was the rate of dissociation of DNA from E·DNA complex, which was about 100-fold slower than the rate of polymerization measured in pre-steady-state assay (Kuchta et al. 1987). How much the hydrogen bonding will affect the rate of polymerization needs to be addressed using pre-steady state kinetic studies. In our model, the geometry of the base pair that controls the $\alpha$-phosphate position of the nucleotide is the key in the control of the fidelity. Although hydrogen bonding may not be as significant as the base-pair geometry itself, it should help to stabilize the base pair in the right geometry for proper catalytic $\mathrm{Mg}^{2+}$ binding and phosphodiester bond formation.

\section{ACKNOWLEDGMENT}

The authors are indebted to Professor K. A. Johnson at Pennsylvania State University for providing us the computer program PSUSIM.

\section{APPENDIX}

Analytical Solution of the Kinetic Scheme. The analytical solution of the observed rate constants of the two phases can be derived as below.

Scheme 1 can be kinetically represented by Scheme 4 .

Scheme 4

$$
\begin{aligned}
&\left(\mathrm{E} \cdot \mathrm{D}_{n} \underset{k_{-1}}{\stackrel{k_{1}[\mathrm{~N}]}{\rightleftharpoons}} \mathrm{E} \cdot \mathrm{D}_{n} \cdot \mathrm{N}\right) \underset{k_{-2}}{\stackrel{k_{2}}{\rightleftharpoons}}\left(\mathrm{E}^{\prime} \cdot \mathrm{D}_{n} \cdot \mathrm{N} \underset{k_{-3}}{\stackrel{k_{3}[\mathrm{M}]}{\rightleftharpoons}} \mathrm{E}^{\prime} \cdot \mathrm{D}_{n} \cdot \mathrm{N} \cdot \mathrm{M}\right) \underset{k_{-4}}{\stackrel{k_{4}}{\rightleftharpoons}} \\
&\left(\mathrm{E}^{\prime \prime} \cdot \mathrm{D}_{n} \cdot \mathrm{N} \cdot \mathrm{M} \underset{k_{-5}}{\stackrel{k_{5}}{\rightleftharpoons}} \mathrm{E}^{\prime \prime} \cdot \mathrm{D}_{n+1} \cdot \mathrm{P} \cdot \mathrm{M}\right)
\end{aligned}
$$

where each pool of species in rapid equilibrium are parenthesized.

Scheme 4 can be further simplified to Scheme 5 .

Scheme 5

$$
\begin{aligned}
\left(\mathrm{E} \cdot \mathrm{D}_{n}+\mathrm{E} \cdot \mathrm{D}_{n} \cdot \mathrm{N}\right) \underset{k_{-2}^{\prime}}{\stackrel{k_{2}^{\prime}}{\rightleftharpoons}}\left(\mathrm{E}^{\prime} \cdot \mathrm{D}_{n} \cdot \mathrm{N}+\mathrm{E}^{\prime} \cdot \mathrm{D}_{n} \cdot \mathrm{N} \cdot \mathrm{M}\right) \underset{k_{4}^{\prime}}{\stackrel{k_{4}^{\prime}}{\rightleftharpoons}} \\
\left(\mathrm{E}^{\prime \prime} \cdot \mathrm{D}_{n} \cdot \mathrm{N} \cdot \mathrm{M}+\mathrm{E}^{\prime \prime} \cdot \mathrm{D}_{n+1} \cdot \mathrm{P} \cdot \mathrm{M}\right)
\end{aligned}
$$

where each pool of species in rapid equilibrium is considered as one combined pool, and

$$
\begin{gathered}
k_{2}^{\prime}=\frac{k_{2}[\mathrm{~N}]}{K_{\mathrm{d}, \mathrm{N}}+[\mathrm{N}]} \quad k_{-2}^{\prime}=\frac{k_{-2} K_{\mathrm{d}, \mathrm{M}}}{K_{\mathrm{d}, \mathrm{M}}+[\mathrm{M}]} \\
k_{4}^{\prime}=\frac{k_{4}[\mathrm{M}]}{K_{\mathrm{d}, \mathrm{M}}+[\mathrm{M}]} \quad k_{-4}^{\prime}=\frac{k_{-4}}{1+K_{5}} \\
K_{\mathrm{d}, \mathrm{N}}=\frac{k_{-1}}{k_{1}} \quad K_{\mathrm{d}, \mathrm{M}}=\frac{k_{-3}}{k_{3}} \quad K_{5}=\frac{k_{5}}{k_{-5}}
\end{gathered}
$$


An overall equilibrium constant for step 5 and steps thereafter should replace $K_{5}$ when a more complete scheme is considered.

The above simplification can be seen with the following example for the calculation of the reverse rate for step 2 in Scheme 4 considering $\mathrm{E}^{\prime} \cdot \mathrm{D}_{n} \cdot \mathrm{N}$ and $\mathrm{E}^{\prime} \cdot \mathrm{D}_{n} \cdot \mathrm{N} \cdot \mathrm{M}$ are in rapid equlibrium at any time.

The reverse rate of step 2 in Scheme 1 is as follows:

$$
\begin{aligned}
& =k_{-2}\left[\mathrm{E}^{\prime} \cdot \mathrm{D} \cdot \mathrm{N}\right] \\
& =k_{-2}\left(\left[\mathrm{E}^{\prime} \cdot \mathrm{D} \cdot \mathrm{N}\right]+\left[\mathrm{E}^{\prime} \cdot \mathrm{D} \cdot \mathrm{N} \cdot \mathrm{M}\right]\right) \frac{K_{\mathrm{d}, \mathrm{M}}}{K_{\mathrm{d}, \mathrm{M}}+[\mathrm{M}]}
\end{aligned}
$$

Therefore, in Scheme 5,

$$
k_{-2}^{\prime}=\frac{k_{-2} K_{\mathrm{d}, \mathrm{M}}}{K_{\mathrm{d}, \mathrm{M}}+[\mathrm{M}]}
$$

Scheme 5 can be solved analytically (Johnson 1986, 1992) and gave the two observed rate constants:

$$
\begin{aligned}
k_{\mathrm{obs}, 1 ; 2} & =\frac{\left(k_{2}^{\prime}+k_{-2}^{\prime}+k_{4}^{\prime}+k_{-4}^{\prime}\right)}{2} \pm \\
& \frac{\sqrt{\left(k_{2}^{\prime}+k_{-2}^{\prime}+k_{4}^{\prime}+k_{-4}^{\prime}\right)^{2}-4\left[k_{2}^{\prime}\left(k_{4}^{\prime}+k_{-4}^{\prime}\right)+k_{-2}^{\prime} k_{-4}^{\prime}\right]}}{2}
\end{aligned}
$$

The observed rate constants calculated from the above analytical equation are same as those obtained from computer simulations.

These two observed rate constants can be approximated to

$$
\begin{aligned}
& k_{\mathrm{obs}, 1} \approx k_{2}^{\prime}+k_{-2}^{\prime}+k_{4}^{\prime}+k_{-4}^{\prime} \\
& \approx \frac{k_{2}[\mathrm{~N}]}{K_{\mathrm{d}, \mathrm{N}}+[\mathrm{N}]}+\frac{k_{-2} K_{\mathrm{d}, \mathrm{M}}}{K_{\mathrm{d}, \mathrm{M}}+[\mathrm{M}]}+ \\
& \frac{k_{4}[\mathrm{M}]}{K_{\mathrm{d}, \mathrm{M}}+[\mathrm{M}]}+\frac{k_{-4}}{1+K_{5}}
\end{aligned}
$$

and

$$
\begin{aligned}
& k_{\mathrm{obs}, 1} \approx \frac{k_{2}^{\prime}\left(k_{4}^{\prime}+k_{-4}^{\prime}\right)+k_{-2}^{\prime} k_{-4}^{\prime}}{k_{2}^{\prime}+k_{-2}^{\prime}+k_{4}^{\prime}+k_{-4}^{\prime}} \approx \\
& \frac{k_{2}[\mathrm{~N}]}{\left.\frac{K_{\mathrm{d}, \mathrm{N}}+[\mathrm{N}]}{K_{\mathrm{d}, \mathrm{M}}+[\mathrm{M}]}+\frac{k_{4}[\mathrm{M}]}{1+K_{5}}\right)+\frac{k_{-2} K_{\mathrm{d}, \mathrm{M}}}{K_{\mathrm{d}, \mathrm{M}}+[\mathrm{M}]} \frac{k_{-4}}{1+K_{5}}} \\
& \frac{k_{2}[\mathrm{~N}]}{K_{\mathrm{d}, \mathrm{N}}+[\mathrm{N}]}+\frac{k_{-2} K_{\mathrm{d}, \mathrm{M}}}{K_{\mathrm{d}, \mathrm{M}}+[\mathrm{M}]}+\frac{k_{4}[\mathrm{M}]}{K_{\mathrm{d}, \mathrm{M}}+[\mathrm{M}]}+\frac{k_{-4}}{1+K_{5}}
\end{aligned}
$$

\section{REFERENCES}

Abbotts, J., SenGupta, D. N., Zmudzka, B., Widen, S. G., Notario, V., \& Wilson, S. H. (1988) Biochemistry 22, 901-909.

Ahn, J., Werneburg, B. G., \& Tsai, M.-D. (1997) Biochemistry 36, $1100-1107$
Anderson, K. S., Sikorski, J. A., \& Johnson, K. A. (1988) Biochemistry 27, 7395-7406.

Barshop, B. A., Wrenn, R. F., \& Frieden, C. (1983) Anal. Biochem. 130, 134-145.

Beard, W. A., Osheroff, W. P., Prasad, R., Sawaya, M. R., Jaju, M., Wood, T. G., Kraut, J., Kunkel, T. A., \& Wilson, S. H. (1996) J. Biol. Chem. 271, 12141-12144.

Bloom, L. B., Otto, M. R., Beechem, J. M., \& Goodman, M. F. (1993) Biochemistry 32, 11247-11258.

Bloom, L. B., Otto, M. R., Eritja, R., Reha-Krantz, L. J., Goodman, M. F., \& Beechem, J. M. (1994) Biochemistry 33, 7576-7586.

Casas-Finet, J. R., Kumar, A., Morris, G., Wilson, S. H., \& Karpel, L. (1991) J. Biol. Chem. 266, 19618-19625.

Dahlberg, M. E., \& Benkovic, S. J. (1991) Biochemistry 30, 48354843

Echols, H., \& Goodman, M. F. (1991) Annu. Rev. Biochem. 60, 477-511.

Erie, D. A., Hajiseyedjavadi, O., Young, M. C., \& Hippel, P. H. v. (1993) Science 262, 867-872.

Fersht, A. (1974) Proc. R. Soc. London, Ser. B 187, 397.

Fersht, A. (1985) Enzyme Structure and Mechanism, 2nd ed., W. H. Freeman \& Co., New York.

Frey, M. W., Sowers, L. C., Millar, D. P., \& Benkovic, S. J. (1995) Biochemistry 34, 9185-9192.

Guest, C. R., Hochstrasser, R. A., Sowers, L. C., \& Millar, D. P. (1991) Biochemistry 30, 3271-3279.

Herschlag, D., Picirilli, J. A., \& Cech, T. R. (1991) Biochemistry $30,4844-4854$

Hochstrasser, R. A., Carver, T. E., Sowers, L. C., \& Millar, D. P. (1994) Biochemistry 33, 11971-11979.

Hsieh, J.-C., Zinnen, S., \& Modrich, P. (1993) J. Biol. Chem. 268, 24607-24613

Hunter, W. N., Brown, T., Anand, N. N., \& Kennard, O. (1986) Nature 320, 552-555.

Hunter, W. N., Brown, T., Kneale, G., Anand, N. N., Rabinovich, D., \& Kennard, O. (1987) J. Biol. Chem. 262, 9962-70.

Johnson, K. A. (1986) Methods Enzymol. 134, 677-705.

Johnson, K. A. (1992) Enzymes 20, 1-61.

Johnson, K. A. (1993) Annu. Rev. Biochem. 62, 685-713.

Kraynov, V., Werneburg, B. G., Zhong, X., Lee, H., Ahn, J., \& Tsai, M.-D. (1997) Biochem. J. 323, 103-111.

Kuchta, R. D., Mizrahi, V., Benkovic, P. A., Johnson, K. A., \& Benkovic, S. J. (1987) Biochemistry 26, 8410-8417.

Loeb. L. A., \& Kunkel, T. A. (1982) Аnnu. Rev. Biochem. 52, 42957.

Moran, S., Ren, R. X.-F., Rumney, S., IV, \& Kool, E. T. (1997) J. Am. Chem. Soc. 119, 2056-2057.

Patel, S. S., Wong, I., \& Johnson, K. A. (1991) Biochemistry 30, 511-525.

Pecoraro, V. L., Hermes, J. D., \& Cleland, W. W. (1984) Biochemistry 23, 5262-5271.

Pelletier, H., Sawaya, M. R., Kumar, A., Wilson, S. H., \& Kraut, J. (1994) Science 264, 1891-1903.

Pelletier, H., Sawaya, M. R., Wolfle, W., Wilson, S. H., \& Kraut, J. (1996a) Biochemistry 35, 12742-12761.

Pelletier, H., Sawaya, M. R., Wolfle, W., Wilson, S. H., \& Kraut, J. (1996b) Biochemistry, 35, 12762-12777.

Raney, K. D., Sowers, L. C., Millar, D., \& Benkovic, S. J. (1994) Proc. Natl. Acad. Sci. U.S.A. 91, 6644-6648.

Rittinger, K., Divita, G., \& Goody, R. S. (1995) Proc. Natl. Acad. Sci. U.S.A. 92, 8046-8049.

Ward, D. C., Reich, E., \& Stryer, L. (1969) J. Biol. Chem. 244, $1228-1237$

Werneburg, B. G., Ahn, J., Zhong, X., Hondal, R. J., Kraynov, V., \& Tsai, M.-D. (1996) Biochemistry 35, 7041-7050.

Wong, I., Patel, S. S., \& Johnson, K. A. (1991) Biochemistry 30, 526-537.

BI963181J 